\title{
Does Motivation Really Count for Sales force Performance
}

\section{in Pharmaceutical Industry?}

\author{
Saroj Kumar Sahoo ${ }^{1}$, Dr. Padmalita Routray ${ }^{2} \&$ Dr. Ashok Kumar Dash ${ }^{3}$ \\ ${ }^{1}$ Rajdhani Engineering College, BPUT, Bhubaneswar, Odisha, India, PIN-751017 \\ ${ }^{2}$ F.M. University, Balasore, Odisha, India, PIN-756019 \\ ${ }^{3}$ CBIS, Balasore College of Engineering, Seragarh, Balasore, Odisha, India, PIN-756060 \\ Correspondence: Saroj Kumar Sahoo, Rajdhani Engineering College, BPUT, Bhubaneswar, Odisha, India, \\ PIN-751017. Tel: 91-943-711-1277. E-mail: sahoosaroj78@yahoo.com
}

Received: December 23, 2013

Accepted: January 8, 2014

Online Published: January 20, 2014

doi:10.5430/bmr.v3n2p1

URL: http://dx.doi.org/10.5430/bmr.v3n2p1

\begin{abstract}
The complex whole of internal psychological force is still remained as a mystery for every psychological \& social science researcher and the management practitioners, academician \& researchers are no different from this dimension of social \& organizational development. In this context the current study aims at analyzing the impact of sales force motivation on their selling performance in the pharmaceutical industry. By adopting the explorative factor analysis with 31 items of the questionnaire, nine motivating factors have been explored. Then these factors are put into ordinal regression (as dependant variable is ordinal in nature) with selling performance. The results refers that five factors (ease of completing work, scope of development, career perspective, internal environment \& no pressure feeling) have significant positive impact on selling performance of pharmaceutical sales force.
\end{abstract}

Keywords: Sales force motivation, Performance, Pharmaceutical industry

\section{Introduction}

Self-driven employees are the real blessings to the organizations, which is now a fact of rarest of the rare in most of the industries. This calls for the discussion of organizations' effort to drive the employees as a whole towards their performance, ultimately towards organizational achievements. So the study of motivation forms an integral part of industrial and vocational psychology in which the concepts of need, incentive and attitude are discussed extensively than the concepts of ability and skill (Vroom, 1995). Not only the industries, the academicians \& researchers worried a lot to get a concrete result regarding these aspects. Report of IBM Healthcare and Life Sciences (April 2005), refers that many pharmaceutical companies have embraced variable pay as away to motivate their field force and align behavior with corporate strategies and many of these companies, however, are discovering that their current compensation management systems are inadequate and not providing the results they expected. So their sales force wastes valuable time in scrutinizing \& disputing their paychecks realizing their compensation payments as slow \& inaccurate. To know about employees' preferences of what motivates them could help improving productivity and building success story for the organizations. If motivation is the underlying cause of performance problem, then its solution becomes more complex and challenging (Griffin, 1990). The Indian pharmaceutical industry is currently in the way of growth \& complexity because of downsizing, mergers and takeovers. According to the Hay Group's Pharmaceutical Sales Force Effectiveness Study, co-sponsored by Pharmaceutical Executive, reps in the 90th percentile are earning just 40 percent more than the average performer. Pharmaceutical sector-which employs a total of more than 500,000 people in India across domestic and global firms - is losing at least 30\% of its workforce annually, primarily in sales and marketing, for reasons ranging from low salaries to lack of growth opportunities and a new perception of it being an unstable industry(Unnikrishna, 2008). In this context, driving (motivating) such employees is a big challenge for the organization now and will be remained so in the near future. So far as retention of talents \& organizational targets are concerned, a major dimension in such organizational overall productivity is attributed to the sales force productivity. High productivity in a sales force comes neither naturally nor accidentally. Therefore, motivational studies pertaining to sales force assume importance especially in the pharmaceutical industry. 


\subsection{Research Problem}

One of the motivationally challenged occupations is the field-sales-force. The most frequently discussed question regarding these employees is that "what propels (motivate) the field sales force to perform more \& more?". And the likely answers are salary, incentives, bonus, good work environment, conducive work culture, manager-subordinate relationship and many more in this regard have been explored. But do these factors really act upon the performance of the sales force or do these factors manifest the motivation, which ultimately direct the performance of the sales force? Till now there is very little ground to confirm the answers of these questions in the pharmaceutical industry. Thus the precise problem statement of the current research is being posed as "does motivation has an impact on performance of pharmaceutical sales force?" The underlying objectives of the current research paper are as follows.

\subsection{Objectives of the study}

1) To study various factors affecting the sales force motivation in pharmaceutical industry.

2) To study the impact of motivation on performance of pharmaceuticals sales force.

\section{Research Design \& Methodology}

In the current research work, researchers intended to follow mostly the causative research design. Since a subjective method of analyzing the data will be helpful in identifying the real practice followed by the industry and it is believed that the interpretations of both subjective \& objective analysis that constitute scientific research knowledge will be more meaningful towards the study result. The researchers have also used their own experience, while interacting with the respondents for the better interpretation.

\subsection{Sampling Design}

Stratified random sampling technique is employed to get representative sample. The target population comprises of near about 6000 pharmaceutical frontline sales force and various sales managers at different levels working in Odisha. Initially, five important towns of Odisha were selected, where pharmaceutical sales force work intensively, then, in a proportionate of 1:4 (10 managers \& 40 trainees or sales executives) of sales managers and sales executives, the sample elements have been decided to be included in the sample. Out of 500 distributed questionnaires, $52 \%$ was the response rate and further $50 \%$ (250) questionnaires were perfectly usable for the current research. Out of these questionnaires, 56 questionnaires from managerial levels and 194 questionnaires from frontlines medicine (sales) representatives (in India, these employees are popularly called as MR) \& trainees, build the sample. The Survey Instrument, self administered questionnaires are distributed to pharmaceutical sales force to get the qualitative data about their perception \& attitude towards different dimensions of motivation. Five point Likert scale is used in order to measure the qualitative response of the respondents.

\subsection{Respondents' Profile in the sample}

The demographic profile of the sample respondents has seven parameters and are presented to understand the respondents, i.e., age, education, gender, position, experience, marital status and income. It is found that almost all the respondents belong to male group (only 3 are female) and around 87 percent are young respondents whose ages are all below 35 years. It is observed that most of the respondents $(58 \%)$ are simple graduates and $27 \%$ are post-graduates in their educational qualification. Out of all the respondents, $46.4 \%$ are married and $53.6 \%$ are unmarried. Taking into account position in the hierarchy, $22.4 \%$ are at managerial level and 77.6 are front line sales employees (medicine representatives). Looking at the annual income of the respondents, $62 \%$ of respondents fall in between Rs. 1.00 lakh to Rs. 2.00 lakh. As far as respondents' experience is concerned, $65 \%$ of total respondents are having below 5 years of experience.

\section{Literature Review}

\subsection{Motivation}

Nohria et. al (2008) described that four emotional drives like 'acquire', 'bond', 'comprehend' \& 'defend' are responsible for employees motivation. And corresponding to these drives, four leavers they proposed like 'reward system', 'culture', 'job design' and 'performance management \& resource allocation process' respectively. To activate these leavers, they proposed some actions like the acquire-drive can be fulfilled by sharply distinguishing the good performers from poor performers, tying reward with performance and paying as well as competitors. The bond-drive can be fulfilled by fostering mutual reliance \& friendship between coworkers, building value collaboration \& team work, and encouraging employees to share best practices. The comprehend-drive can be fulfilled by designing the job that has distinct \& important role in the organization, designing the job that is 
meaningful \& foster a sense of contribution to the organization. The defend-drive can be fulfilled by increasing the transparency of all processes, emphasizing their fairness, and building trust by being just $\&$ transparent in granting rewards, assignments and other forms of recognition. By examining the importance of values, attitudes and leadership behaviours in employee work motivation \& performance, Chowdhury and Amin (2001) have shown that employee values \& job attitudes are more instrumental in producing strong intrinsic motivation while leaders' behaviour enhances employee extrinsic motivation. Malik \& Naeem (2009) conducted a survey of 350 pharmaceutical sales force in Pakistan to identify the complex issue of their motivation that results in identifying three top motivators as pay and fringe benefits, job security, promotion opportunities. The age old belief and research finding hold good that pay and fringe benefits as motivators (hygiene factors) could be attributed to the fact that financial incentives, raise in pay and fringe benefits are always linked with performance of sales force ( Bodla and Naeem, 2010). Further Bodla and Naeem (2010) could find out that relationship with supervisor also acts as motivator since he plays a critical role in coaching of his assigned team of pharmaceutical salespersons. Five distinct job factors as 'growth', 'coworkers', 'promotion opportunities', 'reward \& recognition' and 'job security' are found to be the significant predictors of pharmaceutical sales representatives' overall job satisfaction contributing $71 \%$ variability (Bodla and Naeem, 2008). Motivators such as recognition, work itself, growth and promotion opportunities produces job satisfaction whereas absence of hygiene factors such as job security, compensation package, operating procedures, supervision and relationship with co-workers causes job dissatisfaction of pharmaceutical sales employees (Bodla \& Naeem, 2008). Further, Bodla \& Naeem concluded that the presence of hygiene factors is linked with no job dissatisfaction and the absence of motivators is associated with no job satisfaction. This implies that motivators act as satisfiers and the hygiene factors act as dissatisfiers for pharmaceutical sales-force. The important non-financial motivators for the pharmaceutical sales employees can be good relationships with customers including physicians, job security, high ethical standards in my company and job, being well informed and strong products and brands, recognition for my efforts and skills, having power over other people and being the senior representative in the team (Wiese \& Coetzee, 2013). But, competing against others was identified as the least important motivational factors by Wiese \& Coetzee, (2013).

\subsection{Motivation \& performance}

It is judged by canonical correlation analysis that work motivation (including willingness to work, job satisfaction, and organizational commitment) is an efficient predictor of work related behaviors such as absenteeism, number of hours worked, and intentioll to quit (Bjorklund, 2001). There is a negative correlation between positive achievement motivation behaviour (PAMB) and salespersons' extrinsic motivation (Choudhury, 2007). PAMB has produced a significant influence in salespersons' intrinsic motivation and performance. According to the said researcher salespersons believe that to the extent their supervisors exercise achievement motivation behavior, the greater likelihood that intrinsic rewards would be generated for greater performance. Also it has been seen that as compared to extrinsic motivation, intrinsic motivation is shown to produce relatively a greater impact on performance. Further, Choudhury (2007), shows that relationships between positive achievement motivation behaviour (PAMB), intrinsic motivation and performance is significantly positive. Salesperson's level of motivation control was related to salesperson's performance and motivation control was not found to be related to performance in lower selling-knowledge group sales people (Leach et. al, 2005). Harder working but less-experienced sales agents were shown to perform as well as the more experienced but less-motivated colleagues (Bartkus et. al.1994). Several common incentive systems used to increase performance motivation of sales people strongly suggesting that these methods are effective in increasing performance (Jablonsky \& Devries, 1972; Hamner, 1974; Bealty \& Schneir, 1975; Hamner and Hamner, 1968 - Bushardt, 1988). The reliance on continuous reinforcement incentive schedule and various salary plans are particularly effective in bringing new salesperson's performance to an acceptable level and in sustaining these levels of performance (Nord, 1969; Mawhinney \& Mawhinney, 1982; Allon \& Kolko, 1982 Bushardt, 1988). By examining indicators of pharmaceutical sales force performance through sales force motivation strategy perspective, John et. al (2012), could found that financial incentives in terms of salary, bonus and commission are critical for superior sales force performance. However, he concluded that financial reward even being basic and important, it touches upon only one dimension of motivation. Further, John et. al (2012), concluded that salespeople should be involved in setting sales quota, and this will serve as an effective motivating strategy. Finally, John et. al (2012), found that the impact of meeting with sales managers and salespeople on the sales force performance. Neither learning, goal orientation nor intrinsic motivation contributed significant variance in creative performance of the managerial sales force whereas interestingly both factors had their positive role in nurturing creative ideation and its innovative execution for frontline sales persons (Malik et al., 2012). 


\section{Results \& Discussions}

Starting with reliability test (by Cronbach's $\alpha$ ) of the survey instrument was mandatory. Then to reduce the dimension or to build the constructs, factor analysis has been executed separately for the set of questions intended for direct measurement of motivation, indirect measurement of motivation variables. These factors as regressors (independent) has been put for ordinal regression analysis with selling performance as regressand (dependent variables) to know the impact of motivating factors on selling performance.

\subsection{Scale Reliability}

Except the demographic items \& company-specific items, it has been found that the questionnaire is reliable (nearly $90 \%)$ as the reliability statistics for Cronbach's Alpha is 0.898 which is evident from table.

Table 1 . Test of Reliability

\begin{tabular}{|c|c|c|c|c|c|c|c|c|c|}
\hline \multicolumn{4}{|c|}{ Case Processing Summary } & \multicolumn{3}{|c|}{ Scale Statistics } & \multicolumn{3}{|c|}{ Reliability Statistics } \\
\hline & & $\mathrm{N}$ & $\%$ & Mean & Variance & $\begin{array}{c}\text { Std. } \\
\text { Deviation }\end{array}$ & $\begin{array}{l}\text { No. of } \\
\text { Items }\end{array}$ & $\begin{array}{c}\text { Cronbach's } \\
\text { Alpha }\end{array}$ & $\begin{array}{l}\text { No. of } \\
\text { Items }\end{array}$ \\
\hline \multirow[t]{3}{*}{ Cases } & Valid & 247 & 173.90 & 173.90 & 466.991 & 21.610 & 43 & .898 & 43 \\
\hline & Excluded $^{\mathrm{a}}$ & 3 & 1.2 & & & & & & \\
\hline & Total & 250 & 100.0 & & & & & & \\
\hline \multicolumn{10}{|c|}{ a. Listwise deletion based on all variables in the procedure. } \\
\hline
\end{tabular}

To find the dimensions which indirectly explain the sales force motivation, 31 items were factor analysed using principal component analysis \& varimax rotation. With Kaiser Normalization, it found that the sample adequacy is $89 \%(\mathrm{KMO}=0.892)$ and this is significant $(\mathrm{Sig}=0.000)$. From 31 variables, 9 factors have been extracted, which explain $61 \%$ variance. So these nine factors can be assigned with the name as 'ease of completing the work', 'belongingness', 'freedom', 'scope of development', 'career perspective', 'openness', 'internal environment', 'rationality', \& 'no pressure feeling'. Similarly the satisfying factors are 'career development', 'return to the achievement', 'work condition' \& 'accountability'.

Table 2. KMO and Bartlett's Test

\begin{tabular}{llr}
\hline Kaiser-Meyer-Olkin Measure of Sampling Adequacy. & .892 \\
\hline \multirow{3}{*}{ Bartlett's Test of Sphericity } & Approx. Chi-Square & 2617.365 \\
& $\mathrm{df}$ & 465 \\
& Sig. & .000
\end{tabular}

Table 3. Rotated Component Matrix ${ }^{\mathrm{a}}$

\begin{tabular}{|c|c|c|c|c|c|c|c|c|c|}
\hline & \multicolumn{9}{|c|}{ Component } \\
\hline & 1 & 2 & 3 & 4 & 5 & 6 & 7 & 8 & 9 \\
\hline Can express the core value of the company. & & & & & & & .728 & & -.202 \\
\hline $\begin{array}{l}\text { Know, how success is measured in their } \\
\text { companies }\end{array}$ & .256 & .337 & .231 & .459 & & & .230 & & \\
\hline Proud of the own company. & .219 & .540 & & & .246 & & & .351 & \\
\hline Able to see the own future in the present company & .341 & .325 & .352 & & .391 & & & & .260 \\
\hline $\begin{array}{l}\text { Clear \& effective appraisal system for career } \\
\text { development. }\end{array}$ & .371 & .256 & 293 & & .560 & & & & \\
\hline Feel valued own company & & .528 & & & .425 & & & .320 & \\
\hline $\begin{array}{l}\text { Realises that internal image is consistent with } \\
\text { external image. }\end{array}$ & & & & & .780 & .243 & & & \\
\hline Know the unique selling point of the company. & .423 & & & & .483 & & .408 & & .291 \\
\hline Job is understood by every one of the company. & .234 & .490 & .223 & .381 & & & .248 & .253 & \\
\hline No need to compete unfairly present company. & & & & & & & & & -.717 \\
\hline Freely share necessary information within team. & & & & & .322 & 677 & & & \\
\hline Know that there is no secrecy in the team. & .303 & & & & & 692 & & & \\
\hline Proud of being part of department. & .267 & .701 & & & & .250 & & & \\
\hline Really respect the boss. & & .714 & & .215 & & & .213 & & \\
\hline
\end{tabular}


Not threatened by the team mates.

There are opportunities to develop in the company.

Know that poor performance is not tolerated.

Knows that majority of the time spent with

valuable work.

Realises that the meetings are out come oriented.

Finish the work at a reasonable time.

Can refer authorities for their unclear work

Realises that the work suits to the physical

-environment of the present company.

Can leave office when work is completed.

Realises that they are all treated fairly.

Realises that their success is acknowledged

publicly.

Can give constructive critics in the company.

Can express openly the ambition in the company.

Realises that the feelings are asked \& listened.

Not afraid-of making mistake.

Know that there is a rationalisation of cost.

Can access any information for job done in the

company.

$\begin{array}{lllllllll}.301 & .230 & & \mathbf{. 5 5 0} & .214 & & .259 & \\ .370 & .203 & & \mathbf{. 5 1 2} & .341 & & & & \\ & & .254 & \mathbf{. 7 2 6} & & & .239 & & \\ .344 & .271 & & .254 & & . \mathbf{4 1 8} & & .391 \\ .362 & .258 & & .228 & .333 & & \mathbf{. 3 9 4} & .227 \\ .239 & & & .320 & .286 & & & \mathbf{. 4 5 4} \\ . \mathbf{7 4 6} & & & & & & & \\ \mathbf{. 6 2 1} & & .210 & & & & & \\ & & \mathbf{. 5 3 2} & .309 & & .211 & .350 & \\ & .241 & \mathbf{. 6 8 2} & & .238 & & & .200 \\ .324 & & \mathbf{. 6 6 5} & & & & & \\ & & \mathbf{. 5 5 3} & & .422 & & & -.256 \\ \mathbf{. 4 5 0} & .399 & .218 & & & & .201 & \\ & .299 & .259 & & .250 & \mathbf{. 5 2 4} & .309 & \\ .226 & & & & .216 & \mathbf{. 4 7 1} & .460 & \\ .229 & & & & & & . \mathbf{. 7 4} & \\ \mathbf{. 5 4 6} & .251 & & & & & .249 & -.207\end{array}$

Extraction Method: Principal Component Analysis; Varimax Rotation; Kaiser Normalization. a. Rotation converged in 15 iterations.

4.3 Impact of Relevant Motivating Factors on Selling Performance

The nine factors extracted by the above factor analysis, are put in the ordinal regression analysis (as the dependant variable is ordinal in nature) to test the impact of motivating factors on the sales force performance.

Table 4. Test for suitability of the model

\begin{tabular}{|c|c|c|c|c|c|c|c|c|c|c|}
\hline \multicolumn{2}{|c|}{ Model Fitting Information } & \multicolumn{7}{|c|}{ Goodness-of-Fit } & \multicolumn{2}{|c|}{ Pseudo R-Square } \\
\hline Model & $\begin{array}{c}-2 \text { Log } \\
\text { Likelihood }\end{array}$ & Chi-Square & $\mathrm{df}$ & Sig. & & Chi-Square & $\mathrm{df}$ & Sig. & $\begin{array}{l}\text { Cox and } \\
\text { Snell }\end{array}$ & .451 \\
\hline $\begin{array}{l}\text { Intercept } \\
\text { Only }\end{array}$ & 591.492 & & & & Pearson & 1461.525 & 891 & .000 & Nagelkerke & .497 \\
\hline Final & 441.628 & 149.865 & 89 & .000 & Deviance & 437.233 & 891 & 1.000 & McFadden & .251 \\
\hline
\end{tabular}

The Ordinal Regression procedure (also referred to as PLUM) allows building the model that generate predictions, and evaluate the importance of various predictor variables in cases where the dependent (target) variable is ordinal in nature. The significant $(\mathrm{Sig} .=0.000)$ chi-square statistic $(149.865)$ indicates that the ordinal regression model gives a significant improvement over the baseline intercept-only model (table-6). This basically tells that the model gives better predictions than if someone just guessed basing upon on the marginal probabilities for the outcome categories.

In order to see the observed data for its goodness-of-fit to the model. Table-7 that contains Pearson's chi-square statistic for the model and another chi-square statistic based on the deviance refers that the significant $(\mathrm{Sig} .=0.000)$ statistical values, intended to test whether the observed data are consistent or not with the fitted model. Here large significant value (149.865) would conclude that the data and the model predictions are similar and hence refers to be a good model.

Further, in order to in assess the overall goodness of fit of the model, the pseudo r-square measures (table-8) are calculated that attempt to serve the same function as the coefficient of determination in linear regression models-namely, to summarize the proportion of variance in the dependent variable associated with the predictor (independent) variables. For ordinal regression models, these measures are based on likelihood ratios rather than raw residuals. Three different methods are used to estimate the coefficient of determination. First, Cox and Snell's r-square (Cox and Snell, 1989) said that the ordinal regression with categorical outcomes has a theoretical maximum value of less than 1.0. In the current research the concerned value is well behind 1.0. So, overall goodness-of-fit is justified. Second, Nagelkerke (Nagelkerke, 1991) proposed a modification that allows the index to take values in the full zero-to-one range, and the third method refers to McFadden's r-square (McFadden, 1974), which is another 
version, based on the log-likelihood kernels for the intercept-only model and the full estimated model. In the current research the earlier value is 0.497 and the latter value is 0.251 . So again both the values justify the overall model fit.

Table 5. Parameter Estimates

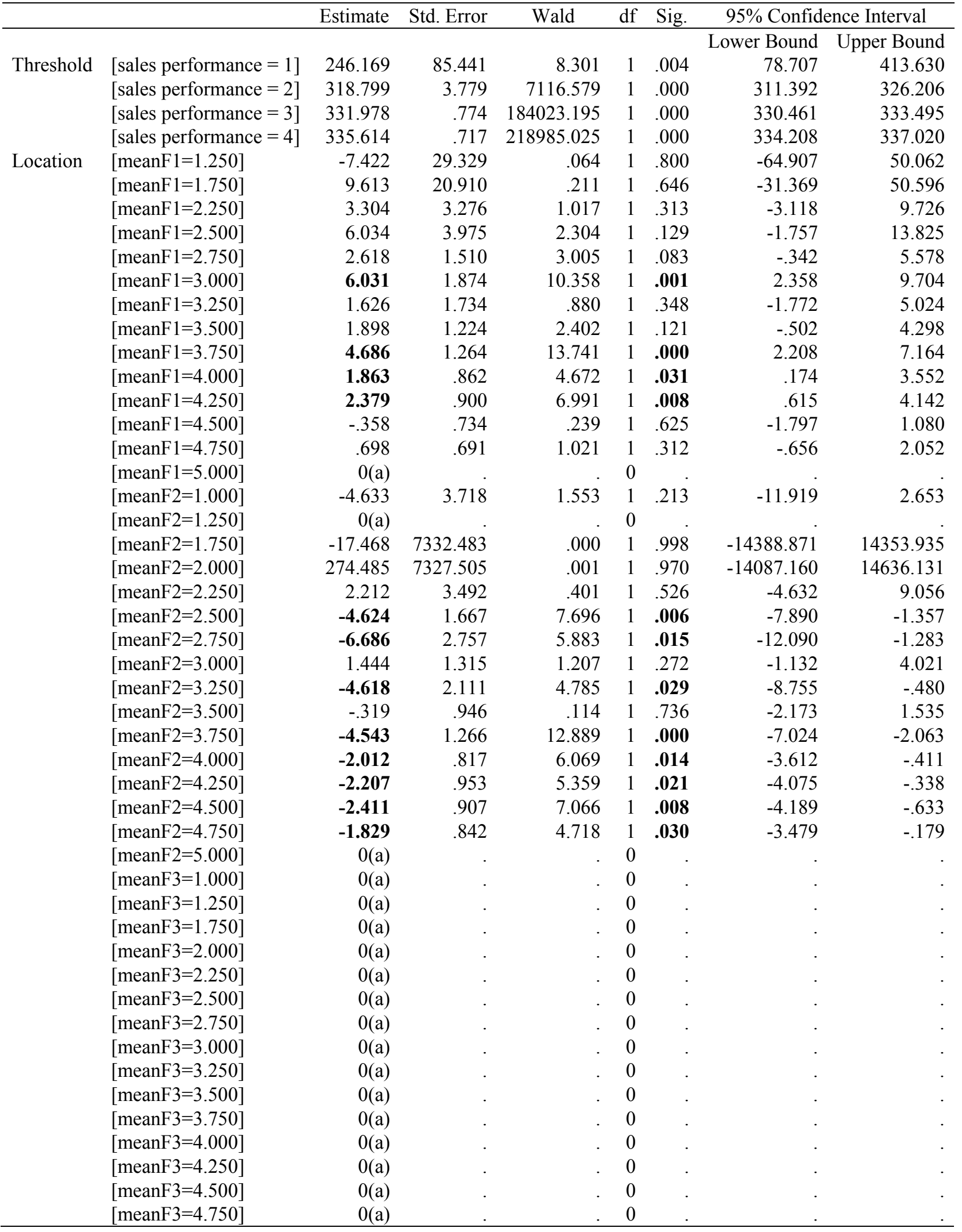




\begin{tabular}{|c|c|c|c|c|c|c|c|}
\hline$[\mathrm{meanF} 3=5.000]$ & 0 (a) & & & 0 & & & \\
\hline$[\mathrm{meanF} 4=1.250]$ & -6.963 & 5.631 & 1.529 & 1 & .216 & -17.999 & 4.074 \\
\hline$[\mathrm{meanF} 4=1.500]$ & -5.627 & 20.573 & .075 & 1 & .784 & -45.950 & 34.696 \\
\hline$[\mathrm{meanF} 4=2.000]$ & -2.542 & 2.179 & 1.361 & 1 & .243 & -6.814 & 1.729 \\
\hline$[\mathrm{meanF} 4=2.250]$ & -2.394 & 2.198 & 1.186 & 1 & .276 & -6.702 & 1.915 \\
\hline$[\mathrm{meanF} 4=2.500]$ & 10.887 & 4.100 & 7.050 & 1 & .008 & -18.924 & -2.851 \\
\hline$[\mathrm{meanF} 4=2.750]$ & 21.420 & 5.026 & 18.165 & 1 & .000 & -31.270 & -11.570 \\
\hline$[\mathrm{meanF} 4=3.000]$ & -.940 & 3.388 & .077 & 1 & .781 & -7.581 & 5.701 \\
\hline$[\mathrm{meanF} 4=3.250]$ & 4.631 & 1.430 & 10.489 & 1 & .001 & -7.434 & -1.828 \\
\hline$[\mathrm{meanF} 4=3.500]$ & 5.005 & 1.388 & 12.992 & 1 & .000 & -7.726 & -2.283 \\
\hline$[$ meanF4 $=3.750]$ & -1.286 & .878 & 2.144 & 1 & .143 & -3.007 & .435 \\
\hline$[\mathrm{meanF} 4=4.000]$ & 2.931 & 1.044 & 7.882 & 1 & .005 & -4.978 & -.885 \\
\hline$[\mathrm{meanF} 4=4.250]$ & 2.130 & .971 & 4.817 & 1 & .028 & -4.033 & -.228 \\
\hline$[\mathrm{meanF} 4=4.500]$ & -.840 & .878 & .916 & 1 & .339 & -2.561 & .881 \\
\hline$[\mathrm{meanF} 4=4.750]$ & -.868 & .718 & 1.462 & 1 & .227 & -2.275 & .539 \\
\hline$[\mathrm{meanF} 4=5.000]$ & $0(\mathrm{a})$ & & . & 0 & . & . & \\
\hline$[$ meanF5 $=1.250]$ & $0(\mathrm{a})$ & & & 0 & & . & \\
\hline$[$ meanF5 $=1.750]$ & -.582 & 20.443 & .001 & 1 & .977 & -40.649 & 39.486 \\
\hline$[$ meanF5 $=2.000]$ & 286.292 & 264.278 & 1.174 & 1 & .279 & -231.684 & 804.268 \\
\hline$[$ meanF5 $=2.250]$ & -4.327 & 3.697 & 1.370 & 1 & .242 & -11.573 & 2.920 \\
\hline$[$ meanF5 $=2.500]$ & 5.530 & 2.200 & 6.321 & 1 & .012 & 1.219 & 9.842 \\
\hline$[$ meanF5 $=2.750]$ & 3.558 & 1.851 & 3.692 & 1 & .055 & -.071 & 7.186 \\
\hline$[$ meanF5 $=3.000]$ & 5.789 & 3.512 & 2.717 & 1 & .099 & -1.094 & 12.672 \\
\hline$[$ meanF5 $=3.250]$ & .584 & 1.181 & .245 & 1 & .621 & -1.730 & 2.899 \\
\hline$[$ meanF5 $=3.500]$ & -.470 & 1.286 & .133 & 1 & .715 & -2.991 & 2.052 \\
\hline$[$ meanF5 $=3.750]$ & 3.354 & 1.017 & 10.888 & 1 & .001 & 1.362 & 5.347 \\
\hline$[$ meanF5 $=4.000]$ & 3.090 & 1.075 & 8.268 & 1 & .004 & .984 & 5.197 \\
\hline$[$ meanF5 $=4.250]$ & 1.998 & .849 & 5.541 & 1 & .019 & .334 & 3.661 \\
\hline$[$ meanF5 $=4.500]$ & 1.790 & .778 & 5.299 & 1 & .021 & .266 & 3.314 \\
\hline$[$ meanF5 $=4.750]$ & .337 & .665 & .257 & 1 & .612 & -.966 & 1.640 \\
\hline$[$ meanF5 $=5.000]$ & $0(\mathrm{a})$ & & & 0 & & & \\
\hline$[$ meanF6 $=1.000]$ & 1.138 & 2.492 & .208 & 1 & .648 & -3.746 & 6.021 \\
\hline$[$ meanF6 $=1.500]$ & -5.887 & 20.302 & .084 & 1 & .772 & -45.678 & 33.905 \\
\hline$[$ meanF6 $=2.000]$ & -6.319 & 3.589 & 3.100 & 1 & .078 & -13.353 & .716 \\
\hline$[$ meanF6 $=2.500]$ & -3.389 & 1.625 & 4.352 & 1 & .037 & .205 & 6.573 \\
\hline$[$ meanF6 $=3.000]$ & -.188 & .853 & .049 & 1 & .825 & -1.861 & 1.484 \\
\hline$[$ meanF6 $=3.500]$ & -2.844 & .887 & 10.283 & 1 & .001 & -4.582 & -1.106 \\
\hline$[\mathrm{meanF} 6=4.000]$ & .005 & .697 & .000 & 1 & .994 & -1.361 & 1.371 \\
\hline$[\mathrm{meanF} 6=4.500]$ & -.772 & .560 & 1.900 & 1 & .168 & -1.870 & .326 \\
\hline$[\mathrm{meanF} 6=5.000]$ & $0(\mathrm{a})$ & & & 0 & & & \\
\hline$[\mathrm{meanF} 7=1.250]$ & 4.468 & 3.139 & 2.026 & 1 & .155 & -1.685 & 10.621 \\
\hline$[$ meanF7 $=1.750]$ & $0(\mathrm{a})$ & & & 0 & & & \\
\hline$[$ meanF7 $=2.000]$ & -1.773 & 2.655 & 446 & 1 & .504 & -6.977 & 3.430 \\
\hline$[$ meanF7 $=2.500]$ & 9.905 & 2.686 & 13.598 & 1 & .000 & -15.170 & -4.640 \\
\hline$[$ meanF7 $=2.750]$ & -2.215 & 2.496 & .787 & 1 & .375 & -7.108 & 2.678 \\
\hline$[\mathrm{meanF} 7=3.000]$ & -1.973 & 3.186 & .383 & 1 & .536 & -8.218 & 4.272 \\
\hline$[\mathrm{meanF} 7=3.250]$ & 1.967 & 1.489 & 1.746 & 1 & .006 & -.951 & 4.886 \\
\hline$[\mathrm{meanF} 7=3.500]$ & 1.253 & 1.033 & 1.470 & 1 & .225 & -.773 & 3.279 \\
\hline$[\mathrm{meanF} 7=3.750]$ & -.482 & .931 & .268 & 1 & .604 & -2.307 & 1.343 \\
\hline$[\mathrm{meanF} 7=4.000]$ & -.389 & .797 & .238 & 1 & .625 & -1.952 & 1.174 \\
\hline$[\mathrm{meanF} 7=4.250]$ & -.442 & .833 & .281 & 1 & .596 & -2.074 & 1.191 \\
\hline$[\mathrm{meanF} 7=4.500]$ & -1.045 & .805 & 1.683 & 1 & .194 & -2.623 & .533 \\
\hline$[$ meanF7 $=4.750]$ & 1.225 & .798 & 2.358 & 1 & .125 & -.338 & 2.789 \\
\hline$[\mathrm{meanF} 7=5.000]$ & 0 (a) & & & 0 & & & \\
\hline$[$ meanF8 $=1.000]$ & 1.298 & 2.678 & .235 & 1 & .628 & -3.951 & 6.547 \\
\hline
\end{tabular}




\begin{tabular}{|c|c|c|c|c|c|c|c|}
\hline$[$ meanF8 $=1.500]$ & -294.991 & 265.059 & 1.239 & 1 & .266 & -814.497 & 224.516 \\
\hline$[\mathrm{meanF} 8=2.000]$ & -5.049 & 1.895 & 7.099 & 1 & .008 & -8.762 & -1.335 \\
\hline$[\mathrm{meanF} 8=2.500]$ & -1.323 & 1.569 & .711 & 1 & .399 & -4.399 & 1.753 \\
\hline$[\mathrm{meanF} 8=3.000]$ & 4.431 & 2.806 & 2.495 & 1 & .114 & -1.068 & 9.930 \\
\hline$[$ meanF8 $=3.500]$ & -3.007 & .970 & 9.616 & 1 & .002 & -4.908 & -1.106 \\
\hline$[$ meanF8 $=4.000]$ & -2.416 & .741 & 10.630 & 1 & .001 & -3.869 & -.964 \\
\hline$[$ meanF8 $=4.500]$ & -.569 & .562 & 1.023 & 1 & .312 & -1.670 & .533 \\
\hline$[\mathrm{meanF} 8=5.000]$ & 0 (a) & & & 0 & & & \\
\hline$[$ meanF9 $=2.000]$ & 340.496 & 7.446 & 2091.091 & 1 & .000 & 325.902 & 355.090 \\
\hline$[$ meanF9 $=2.500]$ & 337.129 & 1.440 & 54801.657 & 1 & .000 & 334.306 & 339.951 \\
\hline$[$ meanF9=3.000] & 334.942 & .827 & 164069.109 & 1 & .000 & 333.321 & 336.562 \\
\hline$[$ meanF9=3.500] & 339.384 & 1.201 & 79886.102 & 1 & .000 & 337.031 & 341.738 \\
\hline$[$ meanF9 $=4.000]$ & 338.015 & .974 & 120399.243 & 1 & .000 & 336.106 & 339.924 \\
\hline$[$ meanF9=4.500] & 334.776 & 637 & 275905.600 & 1 & .000 & 333.527 & 336.025 \\
\hline$[\mathrm{meanF9}=5.000]$ & 334.340 & .000 & . & 1 & • & 334.340 & 334.340 \\
\hline$[$ meanF9 $=23.000]$ & 0 (a) & & & 0 & & . & \\
\hline
\end{tabular}

Link function: Cauchit. a This parameter is set to zero because it is redundant.

Looking at the sig. values and the corresponding estimate values (in bold) from the table of 'parameter estimates'(table-5), it can be concluded that the factor (predictor/ motivator), 'ease of completing work', is a better positive predictor at level $3 \& 4$ of the selling performance. This means for the 'above average' selling performance $\&$ for the 'highest' selling performance, 'ease of completing the work' is a better motivator. Surprisingly at level 4 and to some extent at level $3 \& 2$, the factor, 'belongingness', is a better negative predictor/ motivator for the selling performance. It is Important to note that the factor, 'freedom' has no role in selling performance so far as the current research is concerned. The motivating factor, 'scope of development' is a better positive predictor / motivator both at top levels of selling performance and at lower level selling performance. The factor-5, career perspective, is a better positive motivator at top levels of selling performance. For the average level of selling performance, the factor, 'openness' is a negative predictor at middle levels of selling performance. The factor-motivator, 'internal environment' is a positive predictor of middle level selling-performance. The motivating factor, 'rationality' is significant in some cases as the predictors of selling performance but negatively. Un-doubtedly the last factor, 'no pressure feeling' is a significant positive motivator of selling performance of pharmaceutical sales force.

\section{Summary of Findings}

Through exploratory factor analysis, 9 factors have been extracted from 31 items that are meant for measuring motivation of pharmaceutical sales force and these 9 factors explain 61 percent variance. The names of the motivating factors have been assigned as 'ease of completing the work', 'belongingness', 'freedom', 'scope of development', 'career perspective', 'openness', 'internal environment', 'rationality', \& 'no pressure feeling'

Out of the nine motivating factors, five factors (ease of completing work, scope of development, career perspective, internal environment \& no pressure feeling) have significant positive impact on selling performance of pharmaceutical sales force. Motivating factor (ease of completing work) is a better positive predictor at level $3 \& 4$ of the selling performance. That means for the 'above average' selling performance \& for the 'highest' selling performance, 'ease of completing the work' is a better motivator. Scope of development, is a better positive predictor / motivator both at top levels of selling performance and at lower level selling performance. Career perspective, is a better positive motivator at top levels of selling performance of pharmaceutical sales force. At the middle level of selling performance, internal environment, is a positive predictor (motivator) for the pharmaceutical sales force.

At all level of selling performance, the motivating factors (no pressure feeling) are a strong predictor. Thus, it can be concluded that irrespective of any other perspectives of sales force, if pharmaceutical companies can create such a situation or environment within the work place or within the company, so that their sales people will not feel any pressure to work, then the selling performance can be maintained at least to some extent.

Surprisingly at top level and to some extent at middle level, the factor, 'belongingness', is a better negative predictor/ motivator for the selling performance. Motivating factor, 'open-ness' is a good negative predictor at middle levels of selling performance. And at middle \& lower level of selling performance, 'rationality' is the significant negative predictor for the pharmaceutical sales force. Surprisingly, 'freedom' has no significant role at any level of selling performance of pharmaceutical sales force so far as the current research is concerned

\section{Path for Further Research}

The current research has been mostly oriented towards the impact of motivation on selling performance. The 
interaction of power in the authority hierarchy of sales employees, demographic features, relationship with physicians \& other customers and influence of geographical-areas were assumed to be constant \& favorable, which rarely happen in the pharmaceutical industry. So these variables can be studied further regarding their stake in motivation and performance of pharmaceutical sales force. There may be a chance of effect of intervening variable(s) between sales force motivation and performance. So this can be studied further, which may explore a new horizon of knowledge in managing the sales force.

\section{References}

[Online] Available: http://www.haygroup.com/us/press/details.aspx?id=8848 (press center).

Baart, E., Baranowski , J., Genot, Pierre-Yves, Lee, M., Wynne G. (April 2005). Enhancing pharmaceutical sales force performance: Leveraging variable pay through an enterprise incentive management application, $I B M$ Healthcare and Life Sciences.

Bartkus, Kenneth R., Hartman, Cindy L. \& Parent, C. R. Michel. (1994). The Performance Trade-off Between Experience and Motivation: Its Impact on Travel Service Selling, Journal of Travel Research, 33, 15, pp. 15-20. http://dx.doi.org/10.1177/004728759403300203

Bjorklund, Christina. (2001). Work Motivation- Studies of its Determinants and Outcomes, Dissertation for the degree of Doctor of Philosophy, Stockholm School of Economics, Sweden.

Bodla, Mahmood Ahmad \& Naeem, Basharat. (2008). What Satisfy Pharmaceutical Sales Force in Pakistan, The International Journal of Knowledge, Culture \& Change Management, Volume- 8, November 2, pp. 145-150.

Bodla, Mahmood Ahmad \& Naeem, Basharat. (2010). "Satisfaction \& Dissatisfaction Of Pakistani Pharmaceutical Sales Force", Interdisciplinary Journal Of Contemporary Research Business, Vol. 1, No 12, April.

Bodla, Mohmood A. \& Naeem, Basharat. (2008). Relevance Of Herzberg's Theory To Pharmaceutical Sales Force in Pakistan, International Journal of Knowledge, Culture \& Change Management, Vol. 8, No. 2, pp. 151-158.

Bushardt, Stephen C. Fowler, Aubrey R. Debnath \& Jr. Sukumar. (1988). Sales Force Motivation: A Theoretical Analysis, Human Relations, 41, December, 901- 913. http://dx.doi.org/10.1177/001872678804101202

Chowdhury, Mohammed S. \& Amin, Mohammed Nurul. (2001). Relative Importance of Employee Values, Attitudes and Leadership Behaviours in Employee Motivation: An Empirical Investigation, [Online] Available: http://www.sba.muohio.edu/abas/2001/quebec/chowdhury.pdf

Griffin, R. W. (1990). Management, 3rd edition. Dallas, TX: Houghton Mifflin Company.

John Amue G., Francis Asiegbu I., Chukwu, Ikenna I. (2012). Improving Sales Performance through Sales Force Motivation Strategies: A Study of Pharmaceutical Firms in Nigeria, International Journal of Bussiness Management \& Economic Research, Vol 3(5), pp 620-626.

Leach, Mark P.,Liu, Annie H \& Johnson, Wesley J. (2005). The Role of Self Regulation Training In Developing The Motivation Management Capabilities of Salespeople, Journal of Personal Selling \& Sales Management, Vol. XXV, No. 3 ,Summer, PP 269-281.

Malik Muhammad Ehsan \& Naeem Basharat. (2009). Motivational preferences of Pharmaceutical Salesforce Empirical Evidence From Pakistan, Pakistan Economic And Social Review, Volume 47, No. 1 (Summer 2009), Pp. 19-30.

Mohammed S. Choudhury. (2007). "Enhancing Motivation And Work Performance Of The Salespeople : The Impact Of Supervisors' Behaviour", African Journal Of Business Management, Vol.1 (9), PP. 238-243, December.

Muhammad Ehsan Malik, Basharat Naeem, Waseem Ahmad. (2012). Creative Performance of Pharmaceutical Sales force: Role of Intrinsic Motivation and Learning Goal Orientation, Journal of Basic and Applied Scientific Research, 2(12), pp 12633-12640.

Nitin Nohria, Boris Groysberg, Linda-Eling Lee. (July-August 2008). Employee motivation : A powerful new model, Harvard Business Review, Reprint R0807G.

Unnikrishnan C.H. (2008). Attrition rate among highest in pharma companies, Posted: Mon, Oct 27 2008. 9:14 PM IST, [Online] Available: http://www.livemint.com/2008/10/27205907/Attrition-rate-among-highest-i.html).

Vroom, V. (1995), Work and motivation. In: Wiley, C. (1997), What motivate employees according to 40 years of motivation surveys. International Journal of Manpower, Volume 18(3), pp. 263-280.

Wiese, M., \& Coetzee, R. (2013). The importance of non-financial motivators to pharmaceutical sales representatives: A demographic study, Southern African Business Review, Volume 17, Number 1. 